\title{
Élire des juges et représenter le monde du travail
}

Les élections prud'homales en débats (1907-2014)

Electing Judges and Representing Labour. Debates About Lay Judges Elections

\section{Laurent Willemez}

\section{OpenEdition}

\section{Journals}

Édition électronique

URL : http://journals.openedition.org/travailemploi/6766

DOI : $10.4000 /$ travailemploi.6766

ISSN : 1775-416X

Éditeur

DARES - Ministère du Travail

Édition imprimée

Date de publication : 1 octobre 2015

Pagination : 7-29

ISSN : 0224-4365

Référence électronique

Laurent Willemez, «Élire des juges et représenter le monde du travail », Travail et Emploi [En ligne], 144 | octobre-décembre 2015, mis en ligne le 01 octobre 2017, consulté le 10 décembre 2020. URL : http:// journals.openedition.org/travailemploi/6766 ; DOI : https://doi.org/10.4000/travailemploi.6766 


\title{
Élire des juges et représenter le monde du travail Les élections prud'homales en débats (1907-2014)
}

\author{
Laurent Willemez*
}

\begin{abstract}
Alors que l'élection comme mode de désignation des conseillers prud'hommes a été supprimée par le Parlement, l'article revient sur la manière dont les élections prud'homales ont articulé trois légitimités distinctes, qui construisaient l'identité sociale des conseillers : légitimité syndicale, légitimité juridique et légitimité professionnelle. Pour ce faire, il montre, dans une perspective sociohistorique, comment les débats, et tout particulièrement les débats parlementaires qui ont conduit au vote des deux réformes de 1907 et de 1979, se sont organisés autour de controverses sur les différentes manières de représenter le monde du travail. Il permet ainsi de réinterroger à la fois le concept de « démocratie sociale » et la place occupée par le fait électoral dans le monde du travail.
\end{abstract}

T a juridiction prud'homale est une institution particulièrement originale en France. Sa spécificité principale réside en ce qu'elle a toujours été, au moins jusqu'à ces derniers mois, une justice élue. Cette caractéristique explique en grande partie que cette juridiction ait été considérée comme étant toujours en crise et toujours susceptible de réforme. En effet, ce qui est apparu tout au long du siècle comme une anomalie dans le système judiciaire français, marqué par « l'emprise » de la justice professionnelle (KRYNEN, 2012), est au cœur du questionnement sur la légitimité de l'institution prud'homale et des critiques qu'elle subit : soit parce que, n'étant pas des professionnels du droit, les conseillers prud'hommes ne sont pas assez compétents dans le maniement des outils et des catégories juridiques ; soit parce que, symétriquement, la faiblesse de la participation électorale leur ôte leur représentativité. Si l'analyse de l'activité même de jugement des conseillers conduit à noter combien ils « naviguent » au quotidien entre ces deux écueils, s'efforçant d'être tout à la fois pris au sérieux par les professionnels du droit et reconnus comme des militants syndicaux accomplis (WILLEMEZ, 2012), il ne semble pas inutile de reprendre à nouveaux frais une réflexion

\footnotetext{
* Université de Versailles-Saint-Quentin-en-Yvelines, laboratoire Printemps (Professions, institutions, temporalités ; CNRS-UVSQ, UMR 8085) ; laurent.willemez@uvsq.fr.
} 
sur l'élection prud'homale. Elle nous permettra de nous interroger sur les usages de cette élection dite « sociale » et sur le mode de représentation du monde du travail qu'elle institue.

Pour ce faire, il est nécessaire de revenir à la signification de l'élection dans le cadre prud'homal : l'élection est cet acte social performatif qui donne à des individus le droit de parler et d'agir, ici de juger, au nom d'un groupe social. Ce modèle général est au fondement, comme le montre Patrick Lehingue (2011), de la croyance dans le fonctionnement de la démocratie, et donc au cœur de l'ordre politique. Mais il est aussi efficace dans d' autres univers sociaux, et en particulier dans le monde du travail. Sophie BÉROUD et Karel Yon (2011) montrent ainsi de quelle manière le fait électoral constitue depuis quelques années le point d'appui de la « démocratie sociale » et le moyen tendanciellement exclusif de représentation des salariés, au point que le modèle électoral semble exercer une véritable emprise sur les formes de représentation du monde du travail. La loi de 2008 transformant radicalement les procédures de mesure de la représentativité syndicale est l'aboutissement de ce mouvement (BÉROUD, YoN, 2011). À ce titre, les prud'hommes sont une exception : d'abord parce que leur élection est remise en cause aujourd' hui alors que le fait électoral est l'objet d'une valorisation pour la désignation des autres représentants du monde du travail ; mais, surtout, parce que cette élection a une histoire longue de plus d'un siècle et renvoie à ce que représenter les travailleurs et les patrons veut dire. Depuis le début du Xx ${ }^{e}$ siècle, l'ensemble des débats juridiques, parlementaires et syndicaux consacrés à la modernisation des prud'hommes tournent autour de la question de la « représentation professionnelle ». Alain CHATRIOT (2002) montre combien ces discussions sont, au moins jusqu'en 1939, prises dans un double système d'oppositions : la première distingue, au sein même du monde du travail, une représentation « technique » des principaux acteurs (c'està-dire liée à leur métier) d'une représentation «statutaire » (c'est-à-dire liée à leurs positions de classe); la seconde opposition tient quant à elle à la différence entre représentation de l'intérêt général (pour l'ensemble de la société) et représentation des intérêts spécifiques au monde du travail. Dans cette perspective, les débats sur les élections prud'homales permettent de faire émerger un certain nombre de modèles ou de contre-modèles, qu'il s'agisse des élections politiques, des élections des représentants du personnel dans les entreprises, ou encore de la désignation des représentants syndicaux dans un certain nombre de structures (Conseil national économique, puis Conseil économique et social ; commissions régionales et Commission nationale de conciliation, etc.). Une étude précise des débats conduit tout particulièrement à interroger la place du fait syndical dans l'ensemble de ces instances et le rôle qu'il devrait y occuper : si tout au long du $\mathrm{Xx}^{\mathrm{e}}$ siècle, les syndicats sont censés, comme l'analyse Pierre Rosanvallon (1998), assurer « la représentation sans l'élection », alors comment penser le lien entre la question syndicale et la question électorale ?

L'analyse des discussions sur le mode de représentation permet de mettre en relation les différentes manières de dépeindre le monde du travail, c'est-à-dire de le figurer, d'en dresser le portrait (par exemple à travers l'analyse des rapports de 
force qui y ont cours). Dans son analyse de l'histoire des pratiques culturelles, Roger ChARTIER (2009, p. 90 et suivantes) définit trois aspects du travail de représentation : d'abord un « travail de classement et de découpage qui produit les configurations [...] par lesquelles la réalité est contradictoirement construite par les différents groupes qui composent une société »; ensuite les pratiques sociales qui ont un objectif de « monstration » des identités sociales; enfin le travail politique d'expression, souvent performatif, de l'existence et de la force d'un groupe. Cette décomposition du travail de représentation est utile dès lors que nous souhaitons montrer comment l'activité parlementaire de production des normes électorales (tout comme d'ailleurs, dans un autre registre, le travail statistique [DESROSIÈRES, THÉVENOT, 2002]) est un moment central de production et de mise en scène des catégories, des groupes et des collèges qui constituent le monde du travail.

Une deuxième problématique s'enchâsse à cette première question et renvoie au caractère juridique et judiciaire des conseils de prud'hommes : dans la mesure où les conseillers ont en premier lieu pour fonction de juger au nom du peuple français des litiges individuels entre employeur et salarié, leur connaissance du droit et leurs compétences juridiques sont centrales dans l'activité prud'homale. Mais comment une élection peut-elle venir sanctionner ces compétences et leur donner une légitimité ? Comment l'aspect proprement juridique et judiciaire de leur identité peut-il s'accommoder de cette légitimité élective (WILLEMEZ, 2007) ? Et si le fait électoral - et tout particulièrement son caractère universel - ne peut pas être remis en cause, quels garde-fous peuvent être introduits dans les procédures électorales pour que les juges se voient contraints de respecter l'ordre judiciaire ? Se poser cette question, c'est aussi revenir sur ce que la désignation par l'élection fait aux conseillers et sur la manière dont elle a des effets sur leurs pratiques de jugement.

Les controverses qui alimentent ces débats se donnent à voir d'une manière particulièrement aiguë quand elles se fixent sur les pratiques concrètes de vote, qu'il s'agisse de la constitution des listes électorales, du mode de scrutin, des lieux et des temps du vote. De manière différente dans chaque période, les débats se focalisent sur des éléments qui, alimentant les discussions entre parlementaires, juristes et représentants d'organisations syndicales et professionnelles, butent sur une question qui, toujours, paraît insoluble : quelle est la légitimité des juges prud'homaux, prise entre faits syndical, professionnel et judiciaire ? Cette histoire est scandée par les deux moments lors desquels l'institution prud'homale est réformée de fond en comble : 1907 et 1979. Entre ces dates, et plus particulièrement entre 1907 et le début des années 1970, qui marquent le lancement de la réforme de 1979, les textes juridiques sont très peu nombreux et les discussions ne concernent que très rarement l'élection. 


\section{Redéfinir les contours du salariat pour maîtriser sa représentation politique : les élections dans la réforme de 1907}

Comme l'ont montré nombre d'historiens et de sociologues, les années 1890-1914 sont les années de construction d'un cadre d'intervention étatique dans le monde du travail et dans la vie des individus. Yves CoHen (1995, p. 8) analyse ainsi la manière dont « l'institution progressive d'un véritable droit social [...] [passe par] la construction d'un corpus de règlements, de normes, de lois de "correction préventive", dont le texte du 9 avril 1898 sur les accidents du travail demeure le modèle ». Comme il l'écrit aussi, l'État entre avec une grande prudence dans un processus d'intervention croissante dans le monde industriel et dans l'ensemble de la vie sociale. Son action dans les relations entre employeurs et salariés passe certes par les lois assurant des formes d'hygiène et de sécurité dans les entreprises, mais aussi par l'encadrement des relations entre salariés et employeurs. C'est tout l'objet de la législation industrielle, préfiguration du droit du travail et codifiée à partir de 1910, qui se développe en même temps dans les universités comme un enseignement spécifique et donne lieu à un travail doctrinal à travers tout un ensemble de publications, dont des thèses ${ }^{1}$.

C'est dans ce cadre qu'émergent une attention pour l'institution prud'homale et un mouvement pour sa réforme, qui donnera lieu à plusieurs lois, dont la principale est celle du 27 mars 1907 unifiant la juridiction prud'homale, notamment en l'étendant aux employés - et ainsi à l'ensemble des métiers qui constituent le monde du travail - et aux femmes (voir encadré 1). Jusqu'à cette période, les élections avaient lieu sous le régime des lois de 1848 et 1853 : organisant des votes différents pour les collèges des patrons et des ouvriers, elles accordent l'éligibilité à l'ensemble des ouvriers et intègrent les chefs d'ateliers et les contremaîtres dans le collège des ouvriers. Contrairement à d'autres aspects de la réforme qui transforment profondément l'institution ${ }^{2}$, la loi de 1907 poursuit dans la lignée d'un élargissement du corps électoral qui, comme nous allons le voir, n'en constitue pas moins une évolution considérable.

La réforme a eu le plus grand mal à être adoptée et ces vicissitudes témoignent des réticences face au double élargissement du corps électoral aux employés et aux femmes, tout autant que de l'opposition des parlementaires les plus conservateurs à une institution qui leur paraît remettre en cause l'ordre social traditionnel. En effet, la première proposition de loi réformant l'institution, discutée en 1889, est mise en sommeil plus de dix ans avant d'être adoptée en 1901 par la Chambre des députés, à la suite d'une série de vœux formulés par le Conseil supérieur du travail. Le Sénat adopte la loi, mais en rejetant toutes les modifications du projet votées par la Chambre. Le

1. Le catalogue du Système universitaire de documentation (Sudoc, alimenté par les bibliothèques et centres de documentation de la recherche et de l'enseignement supérieur français) ne référence pas moins de douze thèses de droit soutenues entre 1898 et 1914 sur les conseils de prud'hommes en France.

2. Notamment la définition du taux de compétence des conseils (c'est-à-dire le montant du litige minimal à partir duquel le conseil peut être saisi), la composition du bureau de jugement, ainsi que l'élargissement territorial des conseils. 


\section{ENCADRÉ 1}

\section{Les élections prud'homales après la loi du 27 mars $1907^{1}$}

- L'extension des prud'hommes aux employés conduit à la création de trois types d'électeurs : les électeurs ouvriers, les électeurs employés et les électeurs patrons ;

- le mode d'élection est un scrutin de liste majoritaire à deux tours par catégories ;

- l'élection a lieu le dimanche à la mairie du lieu de travail ;

- les listes électorales sont produites par le maire de chaque commune, assisté d'un électeur ouvrier, d'un électeur employé et d'un électeur patron ;

- le corps électoral est le suivant : l'électorat est élargi aux femmes ; sont électeurs les ouvriers, les employés et les patrons qui ont plus de 25 ans, trois ans d'exercice de la profession et un an de résidence dans le ressort du conseil ; les électeurs doivent enfin être inscrits sur les « listes électorales politiques »;

- les conseillers prud'hommes sont élus pour six ans, et renouvelés par moitié tous les trois ans ;

- les conditions d'éligibilité sont les suivantes : sont éligibles les hommes et les femmes ${ }^{2}$ résidant depuis trois ans dans le ressort du conseil, âgés d'au moins 30 ans, sachant lire et écrire ; sont aussi éligibles les anciens électeurs (autrement dit les retraités) ayant quitté leur profession depuis moins de cinq ans et l'ayant exercée au moins cinq ans dans le ressort ;

- la contestation des élections suit les mêmes principes que ceux indiqués dans la loi de 1883 sur les élections consulaires.

1. Concernant la loi de 1907, les sources consultées pour l'article sont les suivantes : le Bulletin annoté des lois et décrets, 1907, p. 188 et suivantes ; les comptes rendus des débats parlementaires à la Chambre des députés (11, 12 et 14 février 1901) et au Sénat (3 mars, 27 octobre 1904, 29 mars 1905) ; BAFFOs, (1908).

2. C'est une loi légèrement postérieure, celle du 15 novembre 1908, qui élargit aux femmes l'éligibilité.

va-et-vient entre les deux assemblées se poursuit plusieurs années, jusqu'à ce que la menace d'une grève des prud'hommes contraigne le gouvernement à faire voter une première loi (loi du 15 juillet 1905), qui est complétée deux ans après pour obtenir un texte global réformant les prud'hommes. Nous l'avons dit, le projet de loi contient ainsi de nombreux aspects qui ne concernent pas la question de l'élection, mais c'est cette dernière qui est la plus discutée lors des débats parlementaires et qui permet l'expression d'oppositions quant aux enjeux de représentation du monde du travail. C'est bien autour des enjeux de féminisation et d'ouverture aux employés que tournent l'ensemble des débats concernant l'élection.

\section{Comment dépolitiser des élections : expression des classes sociales et «mandat impératif»}

Le cœur des débats relatifs à l'élection prud'homale a porté, et ce jusqu'en 1979, sur la définition des contours de l'électorat, et plus encore sur la question de la multiplicité des corps électoraux. La spécificité du mode de représentation qui structure les 
prud'hommes a en effet toujours contraint à multiplier les corps électoraux et donc les listes électorales, compliquant l'organisation du vote. Puisque les prud'hommes ont été inventés dans une logique quasi corporative et antilibérale de régulation par des pairs des difficultés liées au travail industriel (OLSZAK, 2008), il est logique que les conseillers prud' hommes représentent d'abord leurs mandants. Dès lors, et depuis 1848, les patrons élisent leurs représentants patrons et les ouvriers leurs représentants ouvriers. Cette séparation en deux corps électoraux, conduisant à deux élections différentes bien que se tenant au même moment et dans le même lieu, est perçue dès le milieu du XIX ${ }^{\mathrm{e}}$ siècle comme rendant possible la figuration d'une lutte des classes (DAVID, 1974) ; elle consacre aussi la mise en œuvre d'une "séparation ouvrière », pensée comme l'expression de l'autonomie du monde ouvrier dans le choix de ses représentants (ROSANVALLON, 1998).

À cette première division au sein de l'électorat s'en ajoute une seconde, qui renvoie à une logique de métier, qui est consubstantielle à la prud'homie depuis son invention en 1806. Dans la mesure où les conseillers prud'hommes doivent mobiliser dans leurs jugements une expertise liée à leur activité professionnelle, il est logique que chaque corps électoral soit lui aussi divisé en autant de corps électoraux que de métiers. Cette représentativité, qualifiée de «technique », est d'ailleurs vue comme un contre-feu à la logique classiste et agonistique des prud'hommes : le découpage « vertical» des prud'hommes et la division de l'électorat en catégories professionnelles conduisent à défendre un cadre corporatif à même de lutter contre une division « horizontale » de la société, en particulier en classes. De fait, comme l'écrit Isabelle LESPINET-Moret (2004, p. 358), le « découpage [en "métiers"] opéré sur le réel met en valeur les communautés de travail en ce qu'elles ont de spécifique du point de vue de leur organisation ou de leur activité, insistant sur les persistances du passé et les adaptations à l'époque contemporaine », ce qui conduit à « morceler la question sociale et le monde ouvrier ». Qui plus est, dans les conseils de prud'hommes, le découpage en « catégories » professionnelles ne joue pas véritablement le rôle qui est censé être le sien, et qui consiste à permettre à deux individus en conflit de voir leur dispute arbitrée par un juge partageant la même identité professionnelle. En effet, une même catégorie professionnelle rassemble une variété de métiers distincts. Ainsi que l'explique Louis Cordelet, sénateur de la Sarthe, par ailleurs président d'une caisse régionale du Crédit agricole mutuel, il existe à Paris quatre conseils de prud'hommes distincts et, en leur sein, de multiples catégories :

« De sorte que les justiciables ont une chance sur neuf d'être jugés par des prud'hommes appartenant à leur catégorie. [...] Et même si on est jugé par des prud'hommes de sa catégorie, on peut l'être par des prud'hommes qui n'ont pas plus que vous et moi une compétence spéciale pour statuer sur les différends d'ordre technique. Pourquoi cela ? Parce que dans chaque catégorie il y a un nombre considérable de professions, même des professions essentiellement différentes les unes des autres. »

(Sénat, 27 octobre 1904.) 
Quoi qu'il en soit, ces réflexions sur les manières de penser et de cartographier la société sont logiquement mêlées à des questions politiques, et en particulier à la crainte, partagée par nombre de parlementaires - en particulier des sénateurs - de voir les prud'hommes se transformer en une arène sociale et les élections prud'homales en un moment de lutte politique. Cette volonté d'endiguement à la fois de la politisation des prud'hommes - dont l'élection constitue le nœud - et de leur perception selon une grille classiste se donne clairement à voir dans deux controverses qui nourrissent les débats parlementaires. La première concerne la définition des employés, la seconde le risque du «mandat impératif ».

Comme le montre Christophe CHARLE (1991), la fin du XIX e siècle est marquée par l'extraordinaire essor de la catégorie des employés, qui, pour les contemporains, correspond d'abord aux employés du secteur privé. Ni patrons (puisqu'ils ne sont pas indépendants), ni ouvriers (puisqu'ils exercent des tâches d'organisation et de gestion des hommes et n'ont pas de prise sur la production), les employés jouent ainsi un rôle de «tampon » (CHARLE, 1991, p. 188). Pour autant, il faut distinguer deux types d'employés : d'une part ceux du commerce, et d'autre part ceux de l'industrie et, parmi eux, les contremaîtres qui ne sont certes pas des patrons mais contrôlent tout de même le travail des ouvriers. Dès lors, ouvrir la prud'homie à ces salariés n'appartenant pas à l'industrie ${ }^{3}$, c'est concrétiser, à travers un dispositif institutionnel, l'idée d'une séparation entre les contremaîtres et les patrons. Paul de Beauregard, professeur de droit commercial puis d'économie politique à Paris, député de la Seine, membre du groupe des Républicains progressistes, qui défend l'existence des prud'hommes pour en faire un instrument de paix sociale, semble en prendre son parti :

«La vérité est qu'à notre époque, le chef d'atelier n'est ni patron, ni ouvrier. Il est entre les deux. On comprend fort bien, dès lors, qu'il déplaise à l'ouvrier de voir parmi les électeurs, et au besoin parmi les éligibles ouvriers, un homme auquel, en somme, il obéit, et qui est pour ainsi dire son chef direct. On comprend très bien aussi que le patron ne se soucie pas beaucoup de voir figurer sur la même liste que lui un homme avec lequel il peut demain se trouver en procès. »

(Chambre des députés, 11 février 1901.)

La tripartition de la société française est ainsi d'une certaine manière institutionnalisée par la constitution de trois collèges électoraux différents, comme cela est exprimé dans la loi :

« Sont électeurs ouvriers : les ouvriers, les chefs d'équipe ou contremaîtres prenant part à l'exécution matérielle des travaux industriels et les chefs d'atelier de famille travaillant eux-mêmes ; électeurs employés : les employés de commerce et d'industrie et les contremaitres ne remplissant que des fonctions de surveillance ou de direction ; électeurs patrons : les patrons occupant pour leur compte un ou plusieurs ouvriers ou employés, les associés en nom collectif, ceux qui gèrent ou dirigent pour le compte d'autrui une fabrique, une manufacture, un atelier, un magasin, une mine et

3. Alors que jusqu'ici, les différends qu' ils avaient avec leurs employeurs étaient jugés par les tribunaux de commerce. 
généralement une entreprise industrielle ou commerciale quelconque ; les présidents et membres des conseils d'administration, les ingénieurs et chefs de service tant dans les exploitations minières que dans les diverses industries. »

Cette vision de la société est finalement assez peu discutée, sans doute parce qu'elle renvoie à une réalité que l'on ne peut plus nier ; on comprend alors que le refus par les parlementaires conservateurs, et ce dès les premières discussions de la loi, d'élargir la juridiction aux employés, n'est qu'un prétexte, les prud'hommes étant vus, par les uns et les autres, comme une conquête du mouvement ouvrier. Comme l'écrit Jacques BOUVERESSE (1999, pp. 188-189), « le syndicalisme naissant, tacitement reconnu à la fin du Second Empire, prend appui sur l'institution prud'homale pour s'affermir et élargir son champ d'action ». Ainsi, refuser l'élargissement des prud'hommes, c'est, pour les plus conservateurs, s'efforcer de lutter contre l'essor du syndicalisme. Et dans ce cadre, le principal argument est celui du « mandat impératif ${ }^{4}$ ». On sait que l'un des actes fondateurs du système politique démocratique a été de prohiber, dès 1789 , le mandat impératif pour imposer le mandat représentatif (GAINOT, 2005). Le principe du mandat impératif est alors agité comme une menace perpétuelle par les parlementaires conservateurs, sans que l'on sache vraiment si ce type de mandat est une demande réellement portée par les organisations syndicales et les militants ouvriers à l'endroit des candidats aux élections prud'homales, ou s'il s'agit d'un fantasme conservateur face à la prise de pouvoir des représentants du monde ouvrier. En tout état de cause, cette question revient sans cesse dans les débats, en particulier dans les propos tenus par le rapporteur de la proposition de loi au Sénat, Hippolyte Gay de Savary, sénateur du Tarn. Dans une intervention, il cite ainsi longuement plusieurs exemples de tracts syndicaux exigeant des conseillers un soutien systématique des ouvriers contre les patrons (documents ayant d'ailleurs conduit à l'annulation des élections pour irrégularités). C'est précisément le caractère électif de la désignation des conseillers qui est perçue comme la cause de ce détournement du sens de l'élection :

« La commission s'est très vivement préoccupée de maintenir cette juridiction dans les attributions pour lesquelles elle a été créée, et de ne pas lui donner un développement qui lui ferait perdre son caractère et qui n'est nullement sollicité par l'intérêt et les besoins de la justice. Trop souvent, d'ailleurs, dans le corps électoral, et même dans les conseils de prud'hommes, le rôle de ceux-ci a été étrangement dénaturé. Trop souvent la lutte légitime pour l'élection de conseillers compétents et honnêtes s'est transformée en véritable lutte des classes. Bien plus, les décisions à rendre, qui ne doivent relever que de la conscience du juge, ont été imposées par l'électeur et acceptées par le candidat dans un marché qui n'a rien de commun avec la justice. Le mandat impératif a fait invasion dans un domaine où le candidat ne doit prendre d'autre engagement que de se conduire en juge, après avoir oublié, autant que le permet la nature humaine, les sympathies et les inimitiés du candidat. »

(Sénat, 3 mars 1904.)

4. Qui consiste en réalité à l'imposition par les mandants ou leurs représentants (ici les syndicats) de consignes de vote ou de décisions aux mandataires. 
C'est bien parce que l'élection a nécessairement une «nature » politique, c'està-dire clivante voire agonistique, que les prud'hommes constituent un danger. Il est d'ailleurs étonnant que le principe même de l'élection ne soit pas discuté une seule fois au cours de l'ensemble des débats, comme si la contestation de tout acte électoral était impossible à cette période de la $\mathrm{III}^{\mathrm{e}}$ République. Dans ce moment où le champ politique est en état de consolidation, remettre en cause le principe de l'élection, et tout particulièrement le suffrage universel, désormais tenu pour irréversible pour l'ensemble des assemblées politiques, semble parfaitement impossible. Non seulement aucun débat n'est ouvert formellement sur la légitimité même du fait électoral, mais c'est également le cas pour le mode de scrutin, alors pourtant que les parlementaires discutent à la même période des avantages relatifs des différents modes de scrutin (MARTY, 2006). Dans les débats sur les prud'hommes, l'élection des conseillers au suffrage de tous les salariés et patrons masculins, conquête de 1848 que le Second Empire ne remet pas en cause, reste une évidence, même si la division entre ouvriers et employés d'une part, l'émiettement du corps électoral en catégories d'autre part, contribuent à nuancer le caractère universel du suffrage.

\section{L'éligibilité des femmes entre logiques de représentation et respect de l'ordre des rapports de sexe}

L'entrée des femmes dans le corps électoral puis leur éligibilité sont au cœur des débats parlementaires et des discussions doctrinales au moment de la rénovation de l'institution prud'homale. Il est intéressant de retracer les logiques de ces débats car ils croisent deux séries d'enjeux : d'une part celui de la place des femmes dans le monde du travail, d'autre part celui de l'inégalité des rapports sociaux de sexe.

Il faut prendre la mesure de cette intégration des femmes dans le corps électoral prud'homal : dans la France du début du Xx ${ }^{e}$ siècle, et malgré l'essor du mouvement féministe et des revendications suffragistes, les femmes restent assignées au domaine privé, dans le cadre du modèle des "sphères séparées », donnant au masculin le monopole du public et de la citoyenneté (BERENI et al., 2012, pp. 220-225). L'entrée des femmes aux prud'hommes peut être aussi considérée comme une illustration de la différence de nature entre élections politiques et élections prud'homales, le monde du travail n'apparaissant pas comme un espace politique. Quoi qu'il en soit, même si les débats parlementaires témoignent du sentiment majoritaire quant à l'illégitimité des femmes à sortir de l'espace domestique, leur intégration dans le corps électoral prud'homal s'impose à travers cette loi.

Cela s'explique en particulier par la féminisation du monde du travail. L'intégration des femmes aux prud'hommes est en effet justifiée comme une nécessité entraînée par la transformation structurelle du monde du travail depuis 1890 : la croissance du nombre de femmes dans la population active est spectaculaire, tout particulièrement au sein du salariat : selon le recensement de 1896 étudié par Françoise BATTAGLIOLA (2000), les femmes représentent plus d'un tiers de la population active. En revanche, 
la présence féminine est très inégalement répartie : si elles sont très peu nombreuses dans les secteurs où les travailleurs sont les plus concentrés (notamment dans les plus grands établissements industriels), elles constituent une part très importante des salariés isolés, en particulier dans certains secteurs comme l'industrie des étoffes et du vêtement.

Les promoteurs de la réforme défendent la présence des femmes aux conseils de prud'hommes et leur éligibilité en faisant d'abord valoir l'argument de la représentativité technique puis celui du genre. Ce dernier s'appuie sur des données chiffrées issues du recensement de 1896. La construction d'un argumentaire à partir d'éléments statistiques est d'ailleurs, il faut le noter, une spécificité des débats concernant le monde du travail, dans la mesure où c'est dans ce secteur que s'est développée, à travers l'Office du travail, « la première institution scientifico-administrative » utilisant les ressources d'un appareil statistique et d'enquête (DESROSIÈrES, 1993 ; LESPINETMoRET, 2007). D'autres données sont aussi utilisées dans les débats, comme celles relatives au nombre d'affaires portées devant les conseils de prud'hommes parisiens.

«Les femmes sont admises dans un grand nombre de métiers, non plus seulement comme ouvrières, mais en qualité de directrices de travail. Le rapporteur du projet à la Chambre affirmait que la proportion féminine était de 38,6\% parmi les employeurs et de $31,26 \%$ parmi les travailleurs du commerce et de l'industrie. D'autre part, il ressort d'une statistique dressée par le conseil des prud'hommes des tissus à Paris que la proportion des affaires introduites par les femmes ouvrières ou patrons s'était élevée à $67 \%$ en 1903. Et la nature technique de certains litiges paraissait rendre la présence de l'élément féminin presque indispensable. »

(Bulletin annoté des lois, 1908, p. 638.)

C'est ainsi à partir d'une vision réaliste de la représentation du monde du travail, nécessitée par la perspective d'une représentativité de type technique, qu'est défendue l'éligibilité des femmes. Cet argument est d'ailleurs sans cesse présent parmi les promoteurs d'une égalité des sexes au sein des prud'hommes. Arthur Groussier, député socialiste de la Seine, rapporteur de nombreux projets de lois concernant la protection juridique des femmes et des enfants au travail, explique par exemple :

« Nous demandons qu'on supprime de la loi l'exception qui est formulée contre les femmes. Nous pensons que la femme qui est obligée d'aller à l'atelier a le droit de défendre ses intérêts au même titre que les hommes eux-mêmes et je ne comprendrais pas que l'on voulût empêcher les ouvrières et les femmes employées d'être appelées à choisir, pour juger leurs différends, des personnes de leur sexe sachant beaucoup mieux que les hommes comment et de quelle façon pourront être réglés ces différends. On ne peut pas vraiment prétendre que les femmes soient incapables d'être arbitres dans les questions de travail [...]. Je dirais mieux. Pour certaines professions, les hommes sont absolument incompétents. Il y a des professions qui sont exercées uniquement par des femmes, par exemple la couture, les modes. »

(Chambre des députés, débats du 12 février 1901.) 
Les débats sur le vote et l'éligibilité des femmes renvoient donc, au premier abord, de la même manière que ceux consacrés à la présence des employés, à ce que veut dire représenter le monde du travail. Mais ils renvoient aussi aux « conditions de légitimation des femmes sur la scène électorale » prud'homale (DuLONG, 2003) ou plutôt aux conditions de leur illégitimité. Comme le montre Delphine Dulong s'agissant des élections politiques en 1945, les discours sur l'intégration des femmes dans le corps électoral puis dans l'institution prud'homale elle-même réitèrent des stéréotypes ayant cours à cette période, renvoyant au travail de maintien de l'ordre des rapports sociaux de sexe. De fait, le processus d' « enfermement [des travailleuses] dans leur identité sexuée » (Dulong, 2003, p. 431) se donne à voir à de multiples reprises dans les débats parlementaires comme en dehors des assemblées. Les prises de position sont principalement constituées d'arguments naturalistes associant la femme à la maternité, à la sexualité et au domestique ; c'est à travers l'expression brutale de ces stéréotypes que l'éligibilité des femmes est délégitimée. Émile Chenel, député républicain du Calvados, provoque ainsi l'hilarité de ses collègues en évoquant l'hypothèse d'un couple à la présidence d'un conseil de prud'hommes :

« La Chambre a décidé, mardi dernier, l'éligibilité de la femme et, sans faire de distinction entre la femme libre, maîtresse de ses droits, et la femme engagée dans les liens du mariage, elle a proclamé, d'une façon souveraine et absolue, l'égalité des sexes. À coup sûr, l'électeur sanctionnera un vote aussi galant et mettra en application un principe aussi gracieux (“Très bien !" Et rires). [...] La France aura non seulement ses prud'femmes mais son couple juge. Le conseil des prud'hommes lui aussi ne voudra pas être moins galant que la Chambre, ni moins courtois que l'électeur son maître et, se faisant un point d'honneur de se mettre à la tête du progrès féministe, il élira la femme comme présidente - honneur aux dames (on rit) - ou comme viceprésident - égalité des sexes (nouveaux rires). »

(Chambre des députés, 14 février 1901.)

Derrière ces rires se cache en réalité l'inquiétude des dominants face à la montée du mouvement ouvrier, mais aussi des revendications féminines, montée que l'élection prud'homale matérialise et objective. On la comprend mieux après avoir lu le rapport de la commission de la législation de la chambre de commerce de Lille en 1907, cité in extenso dans sa thèse par Robert BAFFOS (1908, pp. 215-219), pourtant partisan, certes modéré, de l'égalité des femmes aux prud'hommes : il fait en effet montre d'une conception particulièrement patriarcale des rapports sociaux. La chambre de commerce se lamente de ce qu'elle considère « comme une aberration et une perversion du rôle de la femme dans notre société », en particulier parce que cette «innovation » permet «l'ouverture d'un principe qui, d'étape en étape, nous conduirait jusqu'à l'accession de la femme au rôle politique ». Les arguments naturalistes renvoyant la femme au domestique, à la sensibilité et à la fragilité sont utilisés à de nombreuses reprises :

«C'est pour la réserver tout entière à sa mission génératrice de l'humanité, que la loi lui a créé une salutaire subordination sous la protection et la tutelle de l'homme 
qui, lui, a reçu en partage la force, la puissance, la combativité et à qui sont départies toutes les charges de la vie extérieure. »

(Cité par BAFFOS, 1908, p. 216.)

Les débats concernant les élections prud'homales, qui ne constituent qu'un des aspects de la réforme d'ampleur de la juridiction, rendue possible au début du XXe siècle par l'émergence de la question sociale et par la nécessité de produire un cadre juridique pour le monde du travail, permettent donc de comprendre ceux concernant la structuration de la société française et du monde du travail, alors caractérisés par la croissance des classes moyennes et de la place des femmes dans l'ordre social. Ils avalisent en tout cas l'idée selon laquelle l'élection a une fonction de représentation des salariés dans leur ensemble, même si elle reste largement organisée autour de principes issus d'une logique corporative. C'est ainsi prendre acte de l'existence d'une représentation légitime des salariés, qui commence à être assurée par les organisations syndicales et ce même si la politisation inhérente au fait électoral, et donc la production de clivages et de luttes auxquels elle semble conduire inévitablement, constituent une inquiétude majeure de l'élite politique et économique.

\section{Une élection sans enjeu?}

Le cadre de 1907 fixe pour plus de soixante-dix ans le mode opératoire de l'élection prud'homale. Tout au long de la période, au moins jusqu'au début des années 1970, les différentes élections qui ont lieu ne sont guère compétitives, du fait du mode de scrutin et du duopole sur la représentation syndicale constitué de la CGT et, dans une moindre mesure, de la CFTC (avant la scission avec la CFDT $^{5}$ en 1964). En outre, l'organisation même du scrutin (le dimanche à la mairie du lieu de travail) entraîne tout au long de la période une très forte abstention, moins de $10 \%$ des salariés intéressés participant au vote (MCPHERSON, 1962). L'élection sert d'abord à ratifier le choix du (puis des deux) syndicat(s) majoritaire(s).

Dans ces conditions et dans la mesure où elle n'atteint pas le statut de « rite pauvre » pour reprendre la définition que Patrick LEHINGUE (2011, pp. 15-16) donne de l'élection politique, son utilité même pose question. La dimension rituelle du vote n'est ici même pas assurée. Un second élément affaiblit encore le poids de l'élection : la place importante prise par le secrétaire du conseil de prud'hommes. Nommé et rémunéré par la municipalité 6 , il détient presque seul les compétences juridiques lui permettant d'intervenir fortement dans la procédure à travers les rappels de la jurisprudence et parfois, la rédaction des jugements (MCPHERSON, 1962). Finalement, selon Pierre CAM (1981, pp. 81-92), l'élection aurait surtout permis de valoriser une « idéologie de la concorde », principalement du fait de l'indexation des élections sur

5. CGT : Confédération générale du travail ; CFTC : Confédération française des travailleurs chrétiens ; CFDT : Confédération française démocratique du travail.

6. Il n'y a pas systématiquement un conseil par municipalité. Mais lorsqu'il y en a un, le secrétaire est rémunéré par

la municipalité où se trouve le conseil. 
l'activité de travail proprement dite, à travers l'existence de catégories professionnelles fondées sur les logiques de métier. Dans ces conditions, la réforme de 1979 apparaît comme une relégitimation de l'élection qui, nous allons le voir, se fait cependant au prix de son changement de nature.

\section{Produire une élection démocratique dans un cadre syndical : la réforme de 1979}

Réinventés en 1907, les conseils de prud'hommes fonctionnent depuis presque un siècle dans le même cadre juridique et organisationnel. Certes, les ordonnances du 22 décembre 1958 réformant la justice intègrent les conseils de prud'hommes à l'appareil judiciaire. Mais en ce qui concerne l'élection proprement dite, aucune modification n'est mise en œuvre avant les lois de 1979 et 1982. Pour autant, les débats sont nombreux à partir des années 1960 face à ce qui est qualifié de « crise » des conseils de prud'hommes, dont le signe principal serait « l'embouteillage » de la juridiction par un nombre croissant de demandes de justiciables (VAUCHEZ, WILLEMEZ, 2007). Dans ces débats, puis dans la loi de 1979, la question de l'élection se pose d'une manière plus centrale, mais bien différemment de ce qui s'est passé pour la loi de 1907 : l'enjeu n'est plus celui d'une représentativité technique des différents pans du monde du travail, mais celui d'un affrontement potentiel entre une compétence juridique et une représentativité syndicale, dans la perspective d'un processus de rationalisation et d'homogénéisation des différentes cours. Si la réforme de 1979 porte notamment sur des questions de professionnalisation et de normalisation des prud' hommes, celle-ci a principalement pour effet de structurer l'élection autour du fait syndical. L'organisation nouvelle du vote fait ainsi de l'élection un instrument de mesure de l'audience syndicale et un lieu de démocratie sociale. Comme nous le verrons, c'est précisément cette voie qui provoque la remise en cause du processus électoral dans la période actuelle.

\section{Élection ou désignation : des projets de réforme avortés}

Les années 1950 et 1960 voient les premières esquisses de remise en cause de l'élection prud'homale. Même si ces critiques ne concernent pas les procédures électorales elles-mêmes, les décrire permet de définir la matrice des futures attaques dont l'élection est l'objet.

Tout au long du $\mathrm{Xx}^{\mathrm{e}}$ siècle, et en particulier entre les années 1950 et les années 1970, l'institution connaît un double phénomène de judiciarisation et de syndicalisation. La judiciarisation se traduit non seulement par l'intégration des prud'hommes dans l'ordre judiciaire en 1958, mais aussi par l'essor du droit du travail dans sa version universitaire (WILLEMEZ, 2014) comme dans sa version syndicale (WILLEMEZ, 2003), par la présence croissante des avocats comme représentants des parties ou encore par les premiers efforts de formation des conseillers par les organisations syndicales les 
plus puissantes ${ }^{7}$. Dans ces conditions, les débats changent fondamentalement de nature. Ce qui se joue, c'est la compétence juridique des conseillers, qui est souvent opposée à leur légitimité, désormais sous-tendue par leur appartenance syndicale. En 1954, Pierre LAROQUE, conseiller d'État, à l'origine de la loi de 1936 sur les conventions collectives, directeur général de la Sécurité sociale en 1944, devenu en 1964 président de la section sociale du Conseil d'État, publie dans Droit social un article recommandant l'instauration d'une magistrature sociale unifiant sous la direction d'un magistrat de carrière les litiges liés au contrat de travail, aux conflits collectifs de travail et aux assurances sociales. L'échevinage qu'il propose (et qui consiste à faire présider cette nouvelle juridiction par un magistrat de carrière spécialisé dans ces domaines) n'est qu'une étape dans la disparition des juges non professionnels (LAROQUE, 1954). Si la question de l'élection n'est abordée à aucun moment dans l'article ${ }^{8}$, LAROQUE revient sur la critique qui est faite aux juges non professionnels, qui :

« s'ils ont à coup sûr une compétence indiscutable dans les aspects sociaux des questions à résoudre, risquent de n'avoir pas les qualités ordinairement requises d'un juge dont la fonction demeure de dire le droit, d'observer aussi une discipline de forme et de fond qui donne aux justiciables les garanties auxquelles ils ont droit, tout en assurant, par l'unité indispensable de la jurisprudence, l'égalité de tous devant la justice. »

(LAROQUE, 1954, p. 277.)

À notre connaissance, c'est l'une des premières fois que ce topos de faiblesse juridique apparaît dans la critique des conseillers prud'hommes non professionnels et qu'il supplante le thème de leur " politisation ».

Il est particulièrement intéressant de mettre au regard de ce texte de LAROQUE, certes sans lendemain mais très révélateur du questionnement des juristes sur les juges profanes et de la fermeture relative sur la question, un autre projet de réforme des prud'hommes, qui ne ressemble en rien au précédent : émanant en 1964 du Conseil économique et social (CES), ce projet se donne pour point de départ «d'adapter » les règles de fonctionnement de la juridiction à « des conditions sociales en pleine évolution ${ }^{9} \gg$. La crise de l'institution prud'homale est expliquée par une série de phénomènes, parmi lesquels la difficulté d'accès au conseil pour les justiciables, le fait que de nombreux salariés ne relèvent d'aucun conseil et, enfin, la forte abstention aux élections ; ce dernier thème, utilisé pour la première fois, devient la matrice de toutes les justifications futures pour la réforme des élections prud'homales. La solution

7. Que ce soit par des revues et des ouvrages de vulgarisation du droit du travail ou par des séances de formation à proprement parler (HAMELIN, 2008).

8. Pendant cette période, les écrits sur les prud'hommes ne s'intéressent que très faiblement au mode de désignation des conseillers ; et symétriquement, les rares textes consacrés aux élections sociales ne traitent pas des élections prud'homales. Ainsi, un numéro de la Revue française de science politique de 1953 consacré aux «élections sociales » ne cite pas une fois les élections prud'homales mais préfère se concentrer sur les élections aux caisses de Sécurité sociale (cf. notamment VEDEL, 1953).

9. La Réforme de la juridiction prud'homale, avis et rapports du Conseil économique et social, 2 juin 1964. 
envisagée pour résoudre cette crise est radicale : la suppression de l'élection et son remplacement par une procédure de désignation des conseillers prud'hommes par les organisations syndicales et patronales les plus représentatives. Pour la première fois, le principe même de l'élection est remis en cause par une alliance entre les organisations syndicales obtenant les plus faibles résultats aux élections (en particulier Force ouvrière), les représentants du patronat et ceux du monde agricole. Le modèle de représentativité choisi est celui des commissions régionales et de la Commission nationale de conciliation, selon lequel le ministère du Travail établit une «proportionnalité » entre les organisations syndicales ${ }^{10}$. Cet avis du CES reste sans effet, mais il devient l'une des trames à partir desquelles le principe électoral est par lui-même remis en cause.

Les débats resurgissent au cours des années 1970 à la faveur du sentiment d'approfondissement de la crise de l'institution prud'homale et de l'influence renforcée des syndicats, en particulier de la CGT et de la CFDT, qui contribuent désormais à l'agenda des politiques du travail. Avant-projets de loi, colloques associant organisations syndicales de salariés et mouvements de juristes (Syndicat des avocats de France, Syndicat de la magistrature, Association française du droit du travail et de la Sécurité sociale, etc.) se multiplient. Ces prises de position ne concernent qu'en partie la question de l'élection, même si celle-ci suscite bien des clivages. Le principal d'entre eux porte sur la désignation des conseillers : le Conseil national du patronat français (CNPF) et les syndicats minoritaires (Force ouvrière et CFTC en particulier) défendent le remplacement de l'élection par une procédure de désignation, alors que les deux confédérations dominantes militent pour la transformation de la procédure et du système électoraux, avec l'organisation du vote un jour ouvré, l'inscription automatique des salariés sur les listes électorales et surtout, la revendication d'un nouveau mode de scrutin $^{11}$. Face à ces revendications, les gouvernements de droite qui se succèdent au cours des années 1970 semblent hésiter, et les différents avant-projets et projets de loi passent d'une solution à l'autre, sans que l'on comprenne bien la logique de ces évolutions. Ainsi, le dernier avant-projet, présenté aux partenaires sociaux en avril 1976, est « condamné avec vigueur » par la CGT et la CFDT dans un communiqué commun, dans la mesure où « les engagements pris par les ministres concernés ne sont pas tenus : pas d'amélioration des élections, qui conservent leur caractère compliqué, suranné et antidémocratique, absence de financement des frais électoraux, etc. ${ }^{12} »$.

\footnotetext{
10. Plus précisément, le rapport du CES renvoie à la loi du 26 juillet 1957 modifiant la célèbre loi du 11 février 1950 relative aux conventions collectives et aux procédures de règlement des conflits collectifs de travail (c'est cette loi qui rappelle les critères établissant pour les organisations syndicales une présomption irréfragable de représentativité, qui étaient présents dans la circulaire Parodi du 28 mai 1945). La loi de 1957 est elle-même complétée par un décret du 7 janvier 1959 définissant la participation de chaque organisation aux différentes commissions : sans aucune explication, le décret donne six sièges à la CGT, quatre à la CFTC, quatre à Force ouvrière, un à la CGC (Confédération générale des cadres) et un à la Confédération générale des syndicats indépendants.

11. En l'occurrence, un scrutin proportionnel au plus fort reste.

12. Le Monde, 21 avril 1976.
} 
Les deux syndicats majoritaires défendent par ailleurs un « monopole de présentation » des candidats par les organisations syndicales représentatives et la fin des candidatures libres. Ces débats sur l'élection renvoient avant tout à des rapports de force entre organisations et, par conséquent, au caractère désormais principalement syndical de l'élection prud'homale. La réforme de 1979 permet d'avaliser cette conception de l'élection prud'homale comme élément de la démocratie sociale, en laissant de côté les autres débats, qu'ils aient trait à la technicité professionnelle nécessaire à la justice du travail ou à la compétence juridique des conseillers ${ }^{13}$.

\section{La loi de 1979 entre légitimité syndicale et persistance de principes corporatifs}

L'analyse des débats préparatoires à la loi de 1979 permet de revenir sur l'ensemble des questions concernant la représentation du monde du travail et sur la manière dont les différents arguments y sont réagencés. Ces derniers témoignent que l'appartenance syndicale est devenue le seul véritable mode de légitimation des conseillers.

La loi de janvier 1979 (loi n ${ }^{\circ} 79-44$ du 18 janvier 1979) et le décret d'application de mai 1979 « en vue de la première élection des membres des conseils de prud'hommes » (décret ${ }^{0}$ 79-394 du 17 mai 1979) $)^{14}$ constituent une réforme majeure de l'institution prud'homale, en particulier parce qu'ils généralisent et homogénéisent l'institution sur l'ensemble du territoire et qu'ils créent une section propre pour l'encadrement. La loi transforme aussi profondément les procédures électorales ainsi que le mode de scrutin (voir encadré 2). Si elle valide en fin de compte les revendications de la CGT et de la CFDT relatives à l'élection, elle donne lieu à de très vifs débats qui renvoient, une fois de plus, à la question de savoir qui représente véritablement les conseillers prud'hommes. Trois débats, d'inégale importance, illustrent ces interrogations : le premier concerne le monopole de présentation des candidats, le deuxième l'institution d'une section spécifique pour l'encadrement, le troisième la mise en œuvre d'un vote plural, permettant aux grandes entreprises de voter plusieurs fois.

Dès le début des discussions ${ }^{15}$, on comprend que l'élection ne sera pas remise en cause, puisque l'acte électoral est perçu non seulement comme étant l'ingrédient central du principe démocratique (Le Digol, Voilliot, 2011) mais même comme le seul mode de légitimité possible, ainsi que le dit Robert Boulin, alors ministre du Travail et de la Participation :

«La légitimité des juges ne peut se trouver que dans le suffrage universel direct, qui est le système actuel dans les conseils de prud'hommes, ou indirect : désignation des magistrats par le président de la République. [...] Le gouvernement a estimé que l'élection devait être maintenue en substituant au scrutin majoritaire celui de la

13. Ces questions sont abordées dans les débats relatifs au statut et à la formation des conseillers.

14. En 1982, une loi complète le dispositif (notamment, elle organise le statut des conseillers en termes de discipline, de formation, de rémunération, etc., et instaure un Conseil supérieur de la prud'homie) mais ne touche pas aux élections elles-mêmes (sinon pour allonger le mandat des conseillers à cinq ans).

15. Débats à l'Assemblée nationale les 2, 3 et 5 octobre 1978 ; débats au Sénat les 11 et 12 décembre 1978. 
représentation proportionnelle, qui est seule susceptible, en la matière, de permettre une représentation équitable de toutes les tendances du monde du travail. »

(Assemblée nationale, 3 octobre 1978.)

C'est là une manière de reconnaître que la représentation proportionnelle donne à l'élection prud'homale son véritable sens, qui est de représenter les organisations syndicales en fonction de leurs forces réelles, puisqu'elle conduit à une plus grande adéquation entre les votes des électeurs et les sièges obtenus par les organisations. Pour autant, la représentation proportionnelle à la plus forte moyenne favorise, on le sait, les grandes organisations (MARTIN, 1997). Choisir ce mode de scrutin, c'est par ailleurs prendre acte du fait que les élections prud'homales constituent une mesure de l'audience syndicale, ce dont témoignent les commentaires syndicaux, journalistiques et politologiques qui, en 1979, accompagnent les premières élections régies par ce nouveau cadre. Dès lors, l'ensemble des travaux scientifiques consacrés aux élections prud'homales ont pour objet l'analyse des rapports de force syndicaux qu'elles traduisent (ANDOLFATTO, 1992 ; MARTIN, 1983).

\section{ENCADRÉ 2}

\section{Les élections prud'homales après la loi du 11 janvier $1979^{1}$}

- Le scrutin est organisé en collèges (employeurs et salariés) et en sections, au nombre de cinq : industrie, commerce, activités diverses, encadrement et agriculture. Leur instauration entraîne la disparition des «catégories professionnelles » et de la catégorie des employés ;

- le mode de scrutin est la proportionnelle à la plus forte moyenne ;

- l'élection a lieu un jour ouvrable à la mairie du lieu de travail ou dans un local proche ;

- les conseillers prud'hommes sont élus pour cinq ans, et sont tous renouvelés lors d'un même scrutin ;

- les listes électorales, produites par le maire de chaque commune, font l'objet d'un travail préparatoire des entreprises. Celles-ci doivent déclarer les salariés qu'elles emploient, ce qui détermine leur section d'appartenance : l'employeur déclare l'activité principale de l'établissement, qui résulte du numéro de code $\mathrm{APE}^{2}$;

- sont électeurs les salariés ou les employeurs de plus de 16 ans qui exercent une activité professionnelle, sont sous contrat d'apprentissage ou sont au chômage depuis moins de douze mois ;

- sont éligibles les électeurs français inscrits sur les listes électorales, ayant plus de 21 ans et n'ayant pas été condamnés ;

- les règles de contestation sont celles prévues par le Code électoral.

1. Concernant la loi de 1979, outre la loi elle-même, les sources sont notamment BatTut (2002) et ViLLEBRUN, QUÉTANT (1998).

2. Il s'agit du code issu d'une classification de l'Insee (la Nomenclature d'activités françaises [NAF]) qui détermine l'activité principale de l'entreprise. 
Mais le gouvernement ne va pas jusqu'au bout de cette démarche en n'assurant pas le monopole des candidatures aux syndicats représentatifs, comme le revendiquent la CGT et la CFDT et comme le défendent les parlementaires socialistes et communistes dans leurs amendements. Les arguments échangés entre majorité et opposition laissent entrevoir l'existence de débats sur la légitimité des conseillers, qui opposent, certes à de rares moments, représentation syndicale et compétence juridique. Ainsi, Guy Ducoloné, député communiste des Hauts-de-Seine, Jean Foyer, député du Rassemblement pour la république (RPR) du Maine-et-Loire et ancien ministre de la Justice du général de Gaulle, et Gérard Longuet, député de la Meurthe-et-Moselle et rapporteur du projet de loi, ont un débat particulièrement révélateur :

« Guy Ducoloné : Comme nous, les travailleurs savent que, dans les entreprises, seules les organisations représentatives sur le plan national peuvent défendre leurs intérêts.

Jean Foyer : En la circonstance, il ne s'agit pas d'élire des représentants, des délégués qui auraient en quelque sorte la mission de défendre certains intérêts en face d'autres intérêts.

Plusieurs députés communistes et socialistes : Mais si ! [...]

Gérard Longuet : Il s'agit non de mesurer les forces syndicales, ni la représentativité de tel ou tel, mais bien de désigner des juges qui soient à l'image des collèges électoraux qu'ils représentent. »

(Assemblée nationale, 3 octobre 1978.)

Si ces débats montrent avant tout que certaines organisations syndicales et patronales sont bien présentes dans l'hémicycle par le truchement de députés qui se font leurs représentants, ils soulignent également l'incertitude et l'hésitation quant aux fondements de la légitimité des conseillers et au sens à donner à l'élection. En fin de compte, c'est la logique de représentation syndicale qui l'emporte.

Il reste que la réforme de 1979 n'élimine pas totalement un autre mode de représentation puisqu'elle instaure des sections qui reposent sur un découpage large par secteurs d'activité. Ces sections sont présentes aussi bien dans l'organisation des conseils eux-mêmes que lors des élections à travers la composition des collèges électoraux. La loi de 1907 avait créé des sections ${ }^{16}$ qui n'apparaissaient pas dans la procédure électorale et n'avaient qu'une existence fonctionnelle. Les différentes lois n'utilisent d'ailleurs pas ce terme, puisque seule la « catégorie professionnelle » (chaque section rassemblant une multiplicité de catégories professionnelles) distingue à partir de 1907 les salariés et permet d'organiser le vote. Si les auteurs de la loi de 1979 éliminent ce découpage en catégories professionnelles, ils instaurent en revanche un nouveau partage du corps électoral. Et ils font davantage encore, en créant une section de l'encadrement, où juges et justiciables ne sont plus réunis par une proximité de métier mais par une identité de statut au sein de l'entreprise. Ces deux éléments de la réforme renvoient ainsi à un mode de représentation du monde du travail qu'on

16. Industrie et commerce, auxquelles se sont ajoutées la section agriculture en 1932 puis la section des activités diverses en 1958. 
pourrait qualifier d' «infrasyndical » dans la mesure où est restaurée une légitimité corporative s'appuyant sur le métier ou le statut. Les parlementaires de l'opposition ne s'y trompent pas et protestent, au nom de «l'unité du salariat », contre le principe des sections et contre l'existence d'une section de l'encadrement.

Alain Richard, conseiller d'État, député PS du Val-d'Oise, s'oppose ainsi aux sections : il propose que les conseils soient organisés en « chambres » structurées « non plus nécessairement en raison de l'appartenance corporative des justiciables, mais en raison, par exemple, du type de problème à régler ». Un peu plus tard, il rappelle la position des députés socialistes : « Nous restons totalement hostiles à ce système de ségrégation interne au monde des salariés et nous maintenons que la meilleure solution était de donner aux cadres la garantie d'un jugement par leurs pairs à l'intérieur de chambres spécialisées. » Enfin, il précise, concernant les cadres : «Mon ami Paul Quilès a défendu avec suffisamment d'énergie notre position sur le principe de l'unité du salariat pour que nous n'ayons aucune raison de renoncer à cette position », et il demande une nouvelle fois à ce que les cadres soient intégrés pour l'élection à l'ensemble des salariés.

(Assemblée nationale, 3 octobre 1978.)

L'enjeu est alors de dessiner le contour de cette catégorie, c'est-à-dire de réaliser un travail de taxinomie permettant de définir ce que Luc BOLTANSKI (1982, pp. 52-59) appelle « la bonne forme du groupe ». Comme ils l'ont fait pour les employés au début du siècle, les parlementaires cherchent à définir la spécificité des cadres. Celle-ci semble passer par les « responsabilités » et le rôle de « commandement» au sein des entreprises, mais surtout, en dernière analyse, par le niveau de diplôme et la qualification au sens des grilles Parodi et Croizat de 1945 et de la convention collective de 1947 créant une retraite complémentaire des cadres. Ainsi, pour cette catégorie de salariés au moins, la réforme de 1979 met en œuvre un mode de représentation bien spécifique renvoyant à une logique beaucoup plus statutaire que proprement syndicale ${ }^{17}$.

Concernant les élections, un dernier débat, finalement marginal, émerge au moment de la production de la loi, celui du vote plural. Gérard Longuet, le rapporteur du projet de loi à l'Assemblée nationale, propose que dans le collège employeurs, les grandes entreprises disposent d'un nombre de voix supérieur aux entreprises de plus petite taille. L'amendement, rejeté par le gouvernement, l'est aussi par le Sénat au motif qu'il « rappelle dangereusement le vote censitaire [et que] son principe peut apparaître comme une atteinte au principe traditionnel "un homme, une voix" », comme le dit le sénateur centriste de l'Allier, André Rabineau ${ }^{18}$. Il est pourtant voté par l'Assemblée nationale en deuxième lecture. Mais cette disposition est finalement censurée par le Conseil constitutionnel, dans la mesure où elle n'est pas conforme au principe d'égalité

17. Les organisations syndicales ne sont cependant pas totalement absentes de ce débat particulier, comme le montrent les propos du sénateur de Paris, Jean Chérioux : « Je voudrais que M. le ministre nous confirme que la définition des cadres qui se trouve incluse dans le texte a bien reçu l'aval des organisations syndicales de cadres » (Sénat, débats du 11 décembre 1978).

18. Sénat, débats du 11 décembre 1978. 
devant la loi. Le commentaire de cet arrêt insiste sur l'importance de ce principe d'égalité, qui « s'impose avec d'autant plus de force qu'il s'agit d'élire un organe doté d'un pouvoir de décision propre » (FRANCK, 1980, p. 19367). Cette décision, ainsi que les multiples renvois au Code électoral (en particulier pour les contestations), rappelle que l'élection prud'homale reste apparentée aux élections politiques, qui en constituent le modèle principal.

L'existence même de l'élection pour les conseillers prud'hommes conduit le fait prud'homal à être pris en tenaille entre une légitimité syndicale, une légitimité proprement judiciaire et une légitimité due à leur appartenance au monde du travail. C'est ce triple ancrage qui lui donne sa force mais, quand l'un de ces pôles de légitimité s'affaiblit, c'est toute l'institution qui est mise en danger. C'est par exemple ce que montre Françoise SubILEAU (1999) quand elle étudie les élections prud'homales des années 1980 et du début des années 1990, et souligne comment la crise sociale que connaît la France à cette époque déstabilise la présence syndicale mais aussi l'ensemble du tissu économique local et du salariat : c'est d'abord ce contexte économique et social qui provoque la crise de l'institution prud'homale.

Si l'institution est renforcée par le recours à l'élection pour la désignation de ses conseillers, elle se trouve de ce fait même également l'objet de multiples remises en cause. Ainsi depuis le début des années 2000, et depuis plus longtemps encore en réalité $^{19}$, l'élection prud'homale fait l'objet de discussions en raison du coût de son organisation pour les finances publiques et de l'accroissement constant de l'abstention. L'ordonnance de 2004 sur le vote par correspondance, dont on espérait semble-t-il beaucoup, n'a rien changé à cette double problématique. C'est ce qui a amené le gouvernement socialiste à proposer en janvier 2014 une suppression de l'élection et son remplacement par une désignation des conseillers par les organisations reconnues comme représentatives. Le projet s'est d'abord heurté à l'inégalité juridique entre les deux collèges puisque, si les syndicats de salariés peuvent voir leur audience mesurée par voie électorale (depuis la réforme de la représentativité de 2008), il n'en est pas de même pour les organisations patronales, pour lesquelles les conditions de mesure de la représentativité n'ont pas été précisées. C'est chose faite en mars $2014^{20}$ et le ministère du Travail dépose un nouveau projet de loi peu de temps après. Les débats sur ces différents projets, engagés au sein de la commission des affaires sociales de l'Assemblée nationale ${ }^{21}$, y compris lors des auditions des organisations syndicales et patronales, montrent que la représentation des acteurs du monde du travail n'est désormais abordée que sous l'angle de l'abstention (le niveau de participation étant

19. Dès 1987, soit moins de dix ans après sa profonde réforme, Nathalie DoMPNIER (2014) montre la critique dont l'élection prud'homale est l'objet et pointe la volonté de la réformer.

20. Une organisation d'employeurs est représentative si plus de $8 \%$ des entreprises y adhèrent et sont à jour de leur cotisation ; c'est un commissaire aux comptes qui établit la réalité de l'adhésion. La première mesure aura lieu en 2017.

21. Débats à la commission des affaires sociales de l'Assemblée nationale le 29 avril 2014 et à celle du Sénat le 23 juillet 2014. 
considéré comme une mesure de la légitimité). La question du lien professionnel entre justiciable et juge est totalement évacuée de l'ordonnance, de même que celle de la compétence professionnelle de ces juges profanes. C'est ainsi toute la légitimité de l'institution qui semble mise en péril par cette réforme ${ }^{22}$.

Cette réduction du débat à une question de relations professionnelles est sans doute la conséquence non voulue de la réforme de 1979, qui a conduit à faire centralement de l'élection prud'homale (du fait notamment du mode de scrutin) une mesure d'audience de la représentativité syndicale. Dès lors, à partir du moment où les lois de 2008 et 2014 établissent de nouvelles règles pour ces mesures, l'élection est apparue caduque. L'essor de la « démocratie sociale », telle qu'elle émerge dans les années 2000 (BÉROUD, YON, 2011), ne laisse en effet pas de place à l'élection prud'homale : elle ôte à l'institution une bonne partie de son originalité, mais sans régler son déficit de légitimité parmi les juridictions et au sein du monde du travail. En l'absence d'élection, les discours liés à la crise (appuyés sur les indicateurs de rapidité et de stock des jugements) - qui sont utilisés pour légitimer la réforme de l'ensemble des juridictions françaises -, deviennent par conséquent les seuls possibles sur les prud'hommes et font oublier leur spécificité : servir les intérêts des acteurs du monde du travail, autrement dit réguler cet espace en leur nom même.

\section{BIBLIOGRAPHIE}

Andolfatto D. (1992), L'Univers des élections professionnelles : travail et société au crible des urnes, Paris, Éditions ouvrières.

BAFFos R. (1908), La Prud'homie : son évolution, Paris, A. Rousseau.

Battagliola F. (2000), Histoire du travail des femmes, Paris, La Découverte.

BATTUT C. (2002), « Les conseils de prud'hommes : une justice de proximité », Action juridique $C F D T, \mathrm{n}^{\circ} 156$.

Bereni L., Chauvin S., Jaunait A., Revillard A. (2012), Introduction aux études sur le genre, $2^{\mathrm{e}}$ édition, Louvain-la-Neuve, De Boeck.

BéRoud S., Yon K. (coord.) (2011), La loi du 20 août 2008 et ses implications sur les pratiques syndicales en entreprise: sociologie des appropriations pratiques d'un nouveau dispositif juridique, rapport de recherche, convention Dares « Impact des nouvelles règles de la représentativité sur les pratiques et les stratégies syndicales ».

Boltanski L. (1982), Les Cadres : la formation d'un groupe social, Paris, Éditions de Minuit.

BouverESSE J. (1999), « Des élections malgré tout : l'histoire mouvementée des conseils de prud'hommes », in Krynen J. (dir.), L'Élection des juges : essai de bilan historique français et contemporain, Paris, Presses universitaires de France, pp. 165-221.

22. Et ce, d'autant plus que cette loi organique est votée en même temps que la loi Macron, qui réforme fortement les prud'hommes, autant en termes de procédures (qui sont accélérées) qu'en termes de déontologie et de formation (ce qui laisse planer un doute sur les compétences et le sérieux des actuels conseillers prud'hommes). Sur ces réformes, voir WILLEMEZ, 2015. 
CAM P. (1981), Les Prud'hommes, juges ou arbitres? Les fonctions sociales de la justice du travail, Paris, Presses de la Fondation nationale des sciences politiques.

Charle C. (1991), Histoire sociale de la France au XIX ${ }^{e}$ siècle, Paris, Seuil.

ChARTIER R. (2009), Au bord de la falaise : l'histoire entre certitudes et inquiétude, Paris, Albin Michel.

ChAtriot A. (2002), La Démocratie sociale à la française : l'expérience du Conseil national économique, 1924-1940, Paris, La Découverte.

CoHEN Y., BAudouï R. (collab.) (1995), « Introduction générale. Gouverner le social, 18901945 », in Cohen Y., Baudouï R. (dir.), Les Chantiers de la paix sociale : 1900-1940, FontenaySaint-Cloud, ENS éditions, pp. 7-26.

DAVID M. (1974), «L'évolution historique des conseils de prud'hommes en France », Droit social, $\mathrm{n}^{\circ} 2$, pp. 3-21.

Desrosières A. (1993), La Politique des grands nombres : histoire de la raison statistique, Paris, La Découverte.

Desrosières A., ThÉvenot L. (2002), Les Catégories socioprofessionnelles, $5^{\mathrm{e}}$ éd., Paris, La Découverte.

DOMPNiER N. (2014), « Le “bilan largement positif” des élections de 1987. Enjeux politiques de l'organisation et de la contestation d'un scrutin prud'homal », communication au colloque «Histoire(s) d'élection(s) », université Paris Ouest Nanterre.

Dulong D. (2003), « Des actes d'institution d'un genre particulier. Les conditions de légitimation des femmes sur la scène électorale (1945 et 2001) », in Lagroye J. (dir.) La Politisation, Paris, Belin, pp. 425-443.

FrANCK C. (1980), « Conseil constitutionnel. Décision n 78-101 DC. Observations », Semaine juridique (JCP), p. 19367.

GAINOT B. (2005), « Théorie et pratique(s) de la représentation politique », in Martin J.-C. (dir.), La Révolution à l'œuvre : perspectives actuelles dans l'histoire de la Révolution française, Rennes, Presses universitaires de Rennes, pp. 139-149.

HAMELIN D. (2008), «L'ancrage local de la justice du travail : le conseil des prud'hommes de Poitiers 1968-1979 », in Michel H., Willemez L. (dir.), Les Prud'hommes. Actualité d'une justice bicentenaire, Bellecombe-en-Bauges, Éditions du Croquant, pp. 47-69.

KRYNEN J. (2012), L'État de justice. France, XIII $-X X^{e}$ siècle, tome 2. L'emprise contemporaine des juges, Paris, Gallimard.

LARoque P. (1954), « Contentieux social et juridiction sociale », Droit social, pp. 271-280.

Le Digol C., Voilliot C. (2011), « Hors champ. L'analyse politique et les élections professionnelles », in Leclerc O., Lyon-Caen A., L'Essor du vote dans les relations professionnelles : actualités françaises et expériences européennes, Paris, Dalloz, pp. 41-51.

LEHINGUE P. (2011), Le Vote : approches sociologiques de l'institution et des comportements électoraux, Paris, La Découverte. 
LESPINET-Moret I. (2004), « Vers un “corporatisme” républicain ? Les réformateurs de l'Office du travail », in Kaplan S. L., Minard P. (dir.), La France, malade du corporatisme ? XVIII $X X^{e}$ siècles, Paris, Belin, pp. 355-367.

LESPINET-MORET I. (2007), L'Office du travail, 1891-1914 : la République et la réforme sociale, Rennes, Presses universitaires de Rennes.

Martin P. (1983), «Les élections prud'homales de décembre 1982 », Pouvoirs, nº 26, pp. 125-131.

Martin P. (1997), Les Systèmes électoraux et les modes de scrutin, 2 éd., Paris, Montchrestien.

MARTY T. (2006), « La chambre des députés comme ressource et comme théâtre : la consécration de la cause proportionnaliste par le travail en commission du suffrage universel », in Cohen A., Lacroix B., Riutort P. (dir.), Les Formes de l'activité politique : éléments d'analyse sociologique, du XVIII siècle à nos jours, Paris, Presses universitaires de France, pp. 83-101.

McPherson W. (1962), « Les conseils de prud'hommes : une analyse de leur fonctionnement », Droit social, $\mathrm{n}^{\circ} 1$, pp. 19-36.

OlszaK N. (2008), « Invention et défense du caractère paritaire de la justice prud'homal », in Michel H., Willemez L. (dir.), Les Prud'hommes. Actualité d'une justice bicentenaire, Bellecombe-en-Bauges, Éditions du Croquant, pp. 15-24.

Rosanvallon P. (1998), Le Peuple introuvable : histoire de la représentation démocratique en France, Paris, Gallimard.

SubiLEau F. (1999), « Les élections prud'homales. Participation, représentativité, légitimité », Politix, $\mathrm{n}^{\circ} 47$, pp. 151-166.

VAUCHEZ A., WILLEMEZ L. (2007), La Justice face à ses réformateurs, 1980-2006 : entreprises de modernisation et logiques de résistances, Paris, Presses universitaires de France.

Vedel G. (1953), « Comparaison du régime juridique des élections sociales et du régime juridique des élections politiques », Revue française de science politique, vol. 3, n 2, pp. 231-245.

Villebrun J., QuÉTAnt G.-P. (1998), Traité de la juridiction prud'homale, 3 éd., Paris, LGDJ.

WiLLEMEZ L. (2003), « Quand les syndicats se saisissent du droit. Invention et redéfinition d'un rôle », Sociétés contemporaines, $\mathrm{n}^{\circ}$ 52, pp. 17-38.

Willemez L. (2007), « Le sens d'une élection et les frontières de la justice. Les controverses autour des élections prud' homales », in Michel H., Willemez L. (dir.), La Justice au risque des profanes, Paris, Presses universitaires de France, pp. 15-30.

WiLlemez L. (2012), « Les prud'hommes et la fabrique du droit du travail : contribution à une sociologie des rôles judiciaires », Sociologie du travail, vol. 54, nº 1, pp. 112-134.

WiLlEMEZ L. (2014), «Légitimité académique et proximité sociale. Le droit du travail au miroir de ses manuels », in Chambost A.-S. (dir.), Histoire des manuels de droit : une histoire de la littérature juridique comme forme du discours universitaire, Issy-les-Moulineaux, LGDJLextenso éditions, pp. 183-200.

WiLlEMEZ L. (2015), « Les conseils de prud'hommes entre activité judiciaire et logiques syndicales. Histoire et sociologie d'une juridiction non professionnelle (1806-2014) », Les Cahiers de la justice, $\mathrm{n}^{\mathrm{o}} 2$, pp. 157-170. 